Ann. Biol. anim. Bioch. Biophys., I975, 15 (3), 583-588.

\title{
FORMES DE SÉLÉNIUM URINAIRE CHEZ LE MOUTON
}

\author{
M. HIDIROGLOU $\left({ }^{(}\right)$et I. HOFFMAN $\left({ }^{2}\right)$ \\ avec la collaboration technique de R. J. Westerby \\ Agriculture Canada, \\ (Animal Research Institute), \\ Institut de Recherches zootechniques, \\ Ottawa
}

RÉSUMÉ

Le but de ce travail a été de connaître les teneurs ainsi que les formes de sélénium urinaire du mouton à qui l'on a administré cet oligoélément sous différentes formes. Pour chaque forme de sélénium administré, que ce soit, șélénate, sélénite ou sélénométhionine, on s'est servi de deux moutons. La séparation de deux formes de sélénium a été effectuée au moyen d'une colonne à résine anionique, par chromatographie bidimensionnelle sur papier, ou par électrophorèse. Il en ressort que le sélénium, administré sous quelque forme que ce soit, est éliminé rapidement par la voie urinaire, surtout sous forme organique.

\section{INTRODUCTION}

Dans une étude antérieure (HIDIROGlov et coll., r968) nous avons trouvé qu'une partie du sélénium administré au mouton est éliminée par voie urinaire. BYARD (I969) a rapporté que le triméthyle de sélénium était la forme sous laquelle se présentait la majeure partie du sélénium trouvé dans l'urine des petits animaux de laboratoire auxquels on avait administré du sélénite du sodium. PAIMER et coll. (I970) ont retrouvé 25 p. Ioo du sélénium urinaire du rat traité à une forme organique de sélénium sous la forme de triméthyle de sélénium et I 8 p. Ioo sous la forme d'un métabolite non identifié. Le travail rapporté ci-après est une tentative d'isoler les différentes formes de sélénium excrétées dans l'urine du mouton, en employant une nouvelle méthode de chromatographie sur colonne ou encore en se servant de chromatographie sur couches minces et électrophorèse à haut voltage.

(2) Adresse présente : Conseil national de Recherches (Canada). 


\title{
MATÉRIEL, ET MÉTHODES
}

\begin{abstract}
Animaux
On s'est servi de 6 moutons de race croisée Leicester $\times$ Shropshire nourris avec un foin contenant 0,03 p.p.m. de sélénium. Ces animaux ont été placés en cages à métabolisme huit jours avant le début de l'expérience. Tout le matériel, cages, mangeoires, lieux de collection d'urine, etc., était en matériel plastique. Pendant les 5 jours précédent l'administration du sélénium, des prélèvements d'urine ont été effectués chaque matin. Par la suite, après l'administration de sélénium sous forme de sélénite de sodium, de sélénate de sodium ou encore de sélénométhionine, la collection d'urine a continué quotidiennement pendant i 20 heures. Pour chaque forme de sélénium, 2 moutons de poids égal (35-40 $\mathrm{kg}$ environ) ont été traités avec ro $\mathrm{mg}$ de sélénium, l'un par voie orale, l'autre par voie intramusculaire. Des échantillons pondérés quotidiens ont été réalisés à partir des excrétions journalières totales (après homogénéisation), et seulement des urines fraîches ont été utilisées pour la chromatographie sur colonne. En plus, deux moutons de $25 \mathrm{~kg}$, nourris également avec un foin carencé en sélénium, ont reçu par voie intraveineuse $500 \mu \mathrm{Ci}$ de ${ }^{75} \mathrm{Se}$-sélénométhionine.
\end{abstract}

\section{Chromatographie sur conche mince. Electrophorèse en haut voltage.}

L'urine des moutons traités à la 75 Se-sélénométhionine, 24 heures après son administration, a été premièrement déminéralisée sur Amberlite IR- I $20 \mathrm{H}^{+}$puis chromatographiés, soit sur plaque en silice $G$ (AMBERT et coll., I966), ou sur papier Whatman $\mathrm{N}^{0}{ }_{\mathrm{I}}$, en bidimensionnelle d'après la technique de PALmer et coll. (I969) ou encore par électrophorèse en haut voltage, selon REY (1962). On a utilisé par la suite l'autoradiographie en vue d'identifier les taches radioactives.

\section{Technique de Séparation des différentes formes de sélénium. Chromatographie sur colonne.}

Pour la colonne, on a utilisé une burette de $25 \mathrm{ml}$ en pyrex : un tampon de laine de verre a été mis au bas de la colonne que l'on a rempli de résine anionique sous forme de chlorure (Biorad). La résine fut lavée plusieurs fois à l'eau bidistillée jusqu'à obtention d'un liquide parfaitement clair, rinçée 3 fois avec $25 \mathrm{ml}$ d'acide sulfurique $0, \mathrm{IN}$, et relavée 3 fois avec $25 \mathrm{ml}$ d'eau bidistillée. Pour la regénération de la colonne, on a utilisé de l'acide chlorhydrique o, $\mathrm{N}$ suivi par un dernier lavage avec $3 \times 25 \mathrm{ml}$ d'eau bidistillée.

\section{Mode opératoire}

I. Centrifuger $20 \mathrm{ml}$ d'urine pendant Io $\mathrm{mn}$ à $600 \times g$.

2. Prélever le surnageant et le transvaser dans la colonne à chromatographie (CC).

3. Laver les parois intérieures des tubes à centrifugation avec de légères rincées d'eau et transvaser dans la CC. Durant cette opération, éviter de mettre en suspension le sédiment d'urine (vésidu).

4. Rincer la CC 3 fois avec $24 \mathrm{ml}$ d'eau bidistillée. Collecter les éluats de la colonne dans un matras Kjeldahl. Ceci représente la fraction organique.

5. Laver le contenu de la $\mathrm{CC}$ avec $\mathrm{I}$ oo $\mathrm{ml}$ de $\mathrm{SO}_{4} \mathrm{H}_{2} \mathrm{O}$, IN et collecter les éluats dans une fiole volumétrique. Le contenu de cette dernière représente le sélénium total inorganique.

5 a. Prendre $50 \mathrm{ml}$ des éluats acides et les transvaser dans un flacon à micro-Kjeldahl en vue de la minéralisation acide (nitrique, perchlorique et sulfurique) selon la méthode de HofFMAN et coll. (Ig68). Ceci représente la moitié du sélénium inorganique total, lequel a été déterminé selon HoFFMAN et coll. (1968).

5 b. Transvaser dans un flacon Erlenmayer le reste des éluats. Mettre 3 billes de verre, $5 \mathrm{ml}$ d'eau, $5 \mathrm{ml}$ d'acide sulfurique, puis suivre les étapes de la méthode fluorimétrique de détermination du sélénium (HoFFMAN et coll. I 968) et ceci à partir du point où il est question d'ajouter de l'EDTA, de l'hydroxyde d'ammoniaque et de la solution de DAN, laquelle forme un complexe, spécialement avec le sélénite. Il en résulte donc que le sélénium déterminé au paragraphe $5 b$ sans minéralisation acide au préalable représente le sélénium IV (Sélénite).

6. Le sélénium VI (sélénate) a été calculé de la façon suivante $: 2 x$ la quantité de sélénium déterminé dans l'étape $5 a,-2 x$ la quantité de sélénite déterminé dans l'étape $5 b$.

On s'est servi des étalons internes radioactifs (sélénate, sélénite, sélénométhionine) au cours des étajes précitées. Ies quantités récupérées de ces étalons étaient de l'ordre de 95 p. 100 en moyenne. 


\section{RÉSULTATS E'T DISCUSSION}

A l'exception de quelques cas, la fluctuation journalière du sélénium urinaire et ses quantités excrétées furent faibles chez les moutons avant l'administration du sélénium. Cette excrétion de sélénium si basse est probablement le reflet d'une répercution directe du régime carencé en cet élément (tabl. I, 2, 3). L'excrétion urinaire de sélénium était beaucoup plus rapide après administration intramusculaire qu'orale. Lors de l'administration des 3 formes de sélénium par voie intramusculaire, on a remarqué que dans le cas du sélénate ou du sélénite, la moitié du sélénium était excrétée par voie urinaire au bout d'une semaine, tandis que dans le cas de la sélénométhionine, l'excrétion en atteignait le quart seulement. I1 se peut d'ailleurs que l'excrétion de cet acide aminé sélénié soit plus lente que les formes de sélénium inorganique, comme ceci d'ailleurs a été suggéré par JACoBsson et LINDBERG (I968), pour le Mouton, et par Thomson et STEWAR' (I973), pour le Rat.

TABLEAU I

Formes de sélénium (nanogrammes/20 $\mathrm{ml}$ d'urine) urinaive excrétées après l'administration d'une seule dose de $10 \mathrm{mg}$ de sélénite de sodium (IV) par voie orale (O) ou intramusculaive (IM)

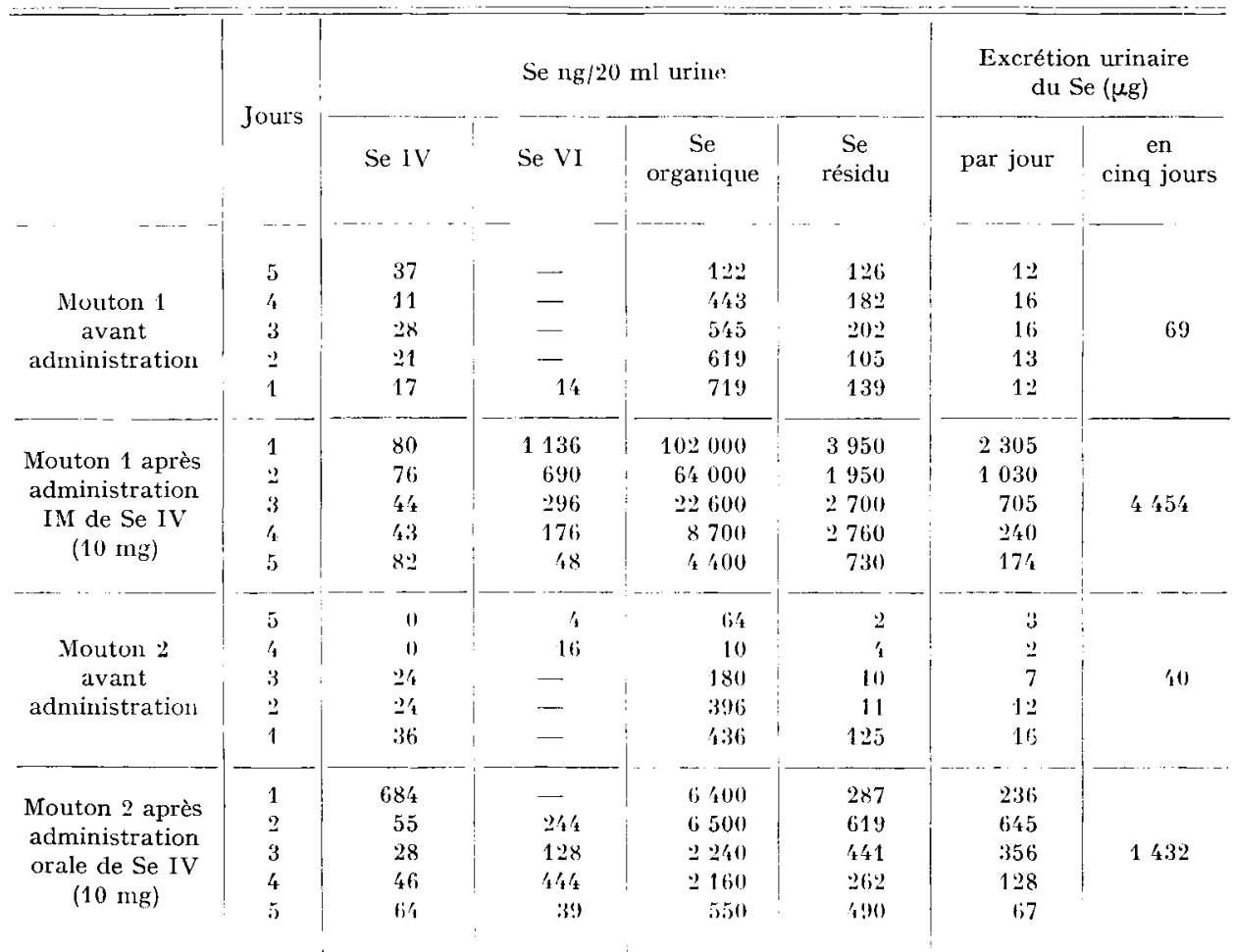

$1 \mu g x \quad 100111 \mathrm{gr}$. 
Après le traitement des moutons au sélénium (tab1. I, 2, 3), les quantités excrétées de cet élément par voie urinaire se rapprochent sensiblement des résultats obtenus chez l'homme, où l'excrétion était de 6,5 à $15 \mathrm{mg}$ de $\mathrm{Se} / 24 \mathrm{~h}$, ceci naturellement de façon comparative par rapport au poids des sujets.

Lors de 1'administration de sélénite ou de sélénate, le sélénium a été davantage excrété au bout d'une semaine dans le cas d'administration IM qu'orale.

Il va de soi que ces observations sont rapportées à titre indicatif, vu le petit nombre d'animaux utilisés. En étudiant les tableaux (I, 2 et 3 ) on constate que la majorité du sélénium excrété avant l'administration de sélénium sous quelque forme que ce soit, est excrété sous forme organique. Une autre partie appréciable se trouve dans le "résiđu », forme de sélénium encore non identifié, puis dans le sélénite. Quant au sélénium rapporté comme « sélénate », il existe en quantité infime.

\section{TABLEAU 2}

Formes de sélénium (nanogrammes/20 $\mathrm{ml}$ d'urine) urinaive excrétées après l'administration d'une seule dose de $10 \mathrm{mg}$ de sélénate de sodium (VI) par voie orale (O) ou intramusculaire (IM)

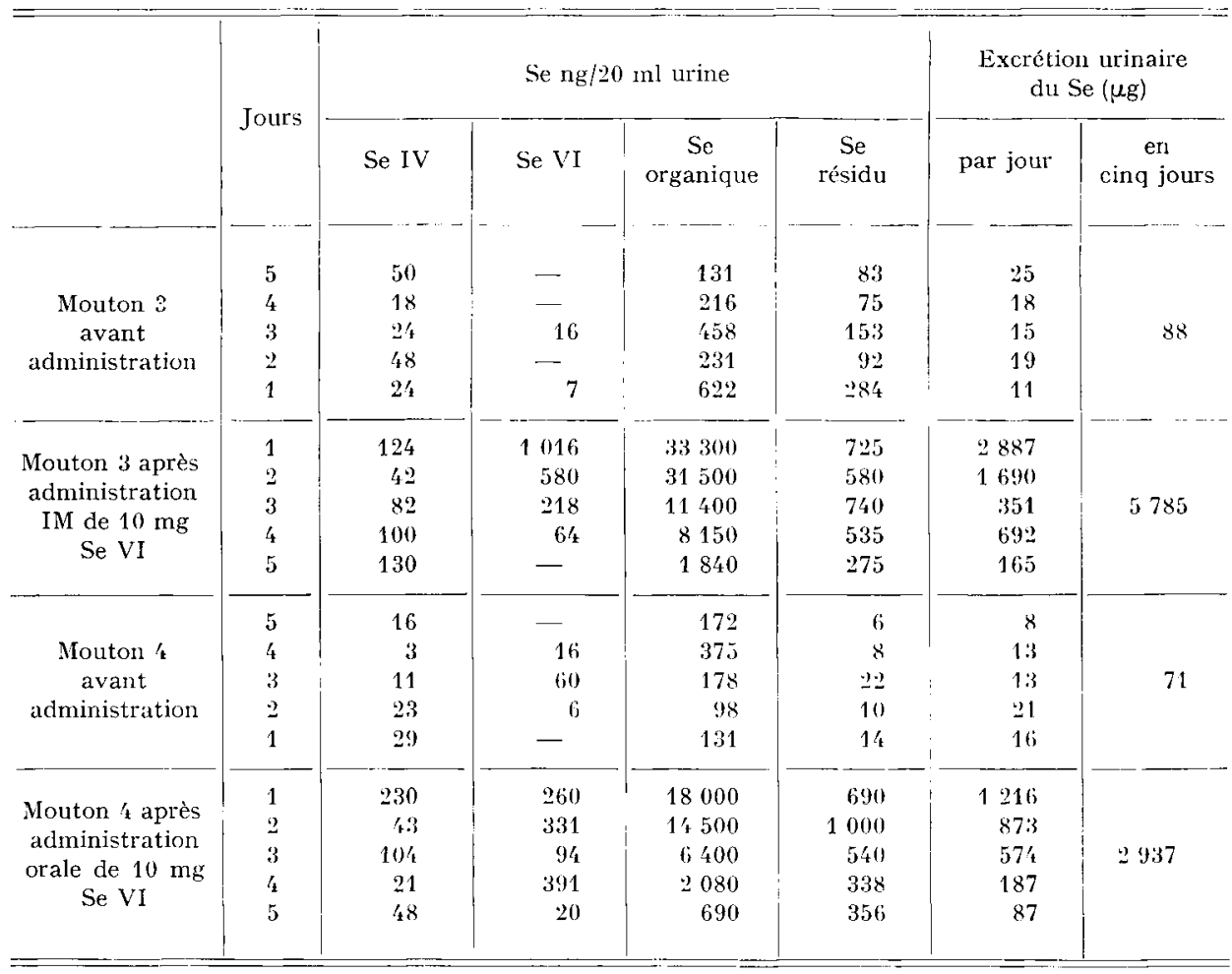

Après déminéralisation de 1'urine du mouton ayant reçu de la ${ }^{75}$ Se-sélénométhionine, sa chromatographie sur couche mince ou électrophorèse à haut voltage suivie d'une autoradiographie de 3 semaines nous ont permis d'isoler une seule tache 
radioactive. Son activité atteignait approximativement 35 p. Ioo de 1'activité originale urinaire échantillonnée.

Il y a lieu de mentionner aussi que la chromatographie bidimensionnelle effectuée d'après la technique de PALMER et coll. (Ig69), où l'on s'est servi de deux systèmes de solvants dans un ordre donné, puis inversé, à confirmé également la présence d'une seule tache radioactive. Cette dernière présentait les même caractères chromatographiques que celles du métabolite organique sélénié UI (le chlorure de triméthyle sélénonium), mentionné par PALMER et Coll. (I969).

\section{TABLEAU 3}

Formes de sélénium (nanogrammes/20 $\mathrm{ml}$ d'urine) urinaire excrétées après l'administration d'une seule dose de $10 \mathrm{mg}$ de sélénométhionine (Se-Me) par voie orale (O) ou intramusculaire (IM)

\begin{tabular}{|c|c|c|c|c|c|c|c|}
\hline & \multirow{2}{*}{ Jours } & \multicolumn{4}{|c|}{ Se $\mathrm{ng} / 20 \mathrm{ml}$ urine } & \multicolumn{2}{|c|}{$\begin{array}{c}\text { Excrétion urinaire } \\
\text { du Se }(\mu g)\end{array}$} \\
\hline & & Se IV & Se VI & $\begin{array}{c}\text { Se } \\
\text { organique }\end{array}$ & $\begin{array}{l}\text { Se } \\
\text { résidu }\end{array}$ & par jour & $\begin{array}{c}\text { en } \\
\text { cing jours }\end{array}$ \\
\hline $\begin{array}{c}\text { Mouton } 5 \\
\text { avant } \\
\text { administration }\end{array}$ & $\begin{array}{l}5 \\
4 \\
3 \\
2 \\
1\end{array}$ & $\begin{array}{r}20 \\
16 \\
45 \\
38 \\
8\end{array}$ & $\begin{array}{l}- \\
- \\
- \\
18\end{array}$ & $\begin{array}{l}204 \\
319 \\
312 \\
403 \\
416\end{array}$ & $\begin{array}{r}98 \\
79 \\
122 \\
72 \\
-\end{array}$ & $\begin{array}{l}18 \\
17 \\
15 \\
12 \\
10\end{array}$ & 72 \\
\hline $\begin{array}{l}\text { Mouton } 5 \text { après } \\
\text { administration } \\
\text { IM de } 10 \mathrm{mg} \\
\text { Se-Me }\end{array}$ & $\begin{array}{l}1 \\
2 \\
3 \\
4 \\
5\end{array}$ & $\begin{array}{l}- \\
90 \\
96 \\
82 \\
96\end{array}$ & $\begin{array}{r}476 \\
222 \\
70 \\
70 \\
-\end{array}$ & $\begin{array}{r}23000 \\
11500 \\
/ 5500 \\
3150 \\
2370\end{array}$ & $\begin{array}{r}1050 \\
680 \\
370 \\
700 \\
500\end{array}$ & $\begin{array}{r}1391 \\
726 \\
26 / \mathbf{k} \\
201 \\
149\end{array}$ & 2731 \\
\hline $\begin{array}{c}\text { Mouton } 6 \\
\text { avant } \\
\text { administration }\end{array}$ & $\begin{array}{l}5 \\
4 \\
3 \\
2 \\
1\end{array}$ & $\begin{array}{r}4 \\
4 \\
5 \\
12 \\
10\end{array}$ & $\begin{array}{r}8 \\
16 \\
11 \\
4 \\
3\end{array}$ & $\begin{array}{l}190 \\
350 \\
396 \\
110 \\
509\end{array}$ & $\begin{array}{l}14 \\
10 \\
16 \\
24 \\
13\end{array}$ & $\begin{array}{r}6 \\
13 \\
9 \\
4 \\
13\end{array}$ & 45 \\
\hline $\begin{array}{c}\text { Mouton } 6 \text { après } \\
\text { administration } \\
\text { orale de } 10 \mathrm{mg} \\
\text { de Se-Me }\end{array}$ & $\begin{array}{l}1 \\
2 \\
3 \\
4 \\
5\end{array}$ & $\begin{array}{l}17 \\
63 \\
40 \\
10 \\
34\end{array}$ & $\begin{array}{r}681 \\
401 \\
248 \\
526 \\
94\end{array}$ & $\begin{array}{r}37000 \\
23500 \\
12000 \\
4500 \\
3130\end{array}$ & $\begin{array}{r}1100 \\
600 \\
550 \\
450 \\
412\end{array}$ & $\begin{array}{r}1051 \\
749 \\
379 \\
209 \\
156\end{array}$ & 2544 \\
\hline
\end{tabular}

\section{CONCLUSION}

Il est évident que le sélénium, indépendamment de la forme sous laquelle il a été administré, est excrété rapidement par la voie urinaire surtout sous sa forme organique. Bien entendu, il serait intéressant de compléter ce travail par une étude similaire sur l'excrétion du sélénium fécal chez le Mouton. 


\section{SUMMARY}

\section{FORMS OF URINARY SEI,ENIUM IN SHEEI'}

The purpose of the present study was to determine the amount and kinds of selenium excretory products in the urine of sheep after administration of this trace element in different forms. Two sheep were allocated to each treatment comprising the administration of one of the following forms of selenium : selenate, selenite and selenomethionine. Two selenium excretory products were separated by means of anion-exchange resins, two-dimensional paper chromatography or by electrophoresis. Results show that selenium, and especially the organic fraction, is rapidly excreted in the urine whatever the form administered.

\section{RÉFÉRENCE,S BIBLIOGRAPHIQUES}

Ambert J.-P., Péchery C., Charpentier C., Hartmann L., ig66. I. Dosage et différenciation des acides aminés urinaire chez le sujet normal. Ann. Biochim. Clin., 24, 17-40.

Byard L. J., 1969. Trimethyl selenide. A urinary metabolite of selenite. Arch. Biochem. Biophys., $130,556-560$.

Hidiroglou M., Jenkins K. J., Carson R. B., Mckay R. R., ig68. Some aspects of selenjum metabolism in normal and dystrophic sheep. Can. J. Animal Sci., 48, 335-346.

Hoffman I., Westerby R., Hidiroglou M., 1968. Precise fluorometric microdetermination in agricultural materials. J.A.O.A.C., 5, ro39-ro42.

JACoBsson S. O., LiNDBERG P., I968. The influence of a flushing dose of selenium on the excretion of selenium in sheep. Acta Vet. Scand., 9, 199-207.

Palmer I. S., Fischer D. D., Halverson A. W., Olson O. E., I969. Identification of a major selenium excretory product in rat urine. Biochim. Biophys. Acta., 197, 336-342.

Palmer I. S., Gunsalus R. P., Halverson A. W., Olson O. E., 197o. Trimethylselenomium ion as a general excretory product from selenium metabolism in the rat. Biochim. Biophys. Acta., 208, 260-266.

Rey J., Mayer M. A., Deysson A., Frézai J., Lamy M., ig62. Séparation bi-dimensionnelle des acides aminés par électrophorèse en haut voltage et chromatographie sur papier. Rev. Franc. Études Clin. et Biol., 7, 877-882.

Thomson D. C., I972. Urinary excretion of selenium in some New Zealand women. Proc. University Otago. Med. School., 50, 3 I-33.

Thomson C. D., Stewart R. D., I973. Metabolic studies of ${ }^{75}$ Se-selenomethionine and ${ }^{75}$ Se-selenite in the rat. Br. J. Nutr., 30, I39-I 47 . 